Published in PRL, 111, 053201 (2013)

\title{
Quantum tunneling of oxygen atoms on very cold surfaces
}

\author{
M. Minissale ${ }^{*}$ E. Congiu, S. Baouche, H. Chaabouni, A. Moudens, and F. Dulieu \\ Université de Cergy Pontoise and Observatoire de Paris, \\ ENS, UPMC, UMR 8112 du CNRS \\ 5, mail Gay Lussac, 95000 Cergy Pontoise cedex, France. \\ M. Accolla \\ Dipartimento di Scienze Applicate, Università degli Studi di Napoli Parthenope, \\ Centro Direzionale Isola C4, 80143 Napoli, Italy. \\ S. Cazaux \\ Kapteyn Astronomical Institute, PO box 800, \\ 9700AV Groningen, The Netherlands. \\ G. Manicó and V. Pirronello \\ Dipartimento di Fisica e Astronomia, Università di Catania, \\ via Santa Sofia 64, 95123 Catania, Sicily, Italy.
}

\begin{abstract}
Any evolving system can change of state via thermal mechanisms (hopping a barrier) or via quantum tunneling. Most of the time, efficient classical mechanisms dominate at high temperatures. This is why an increase of the temperature can initiate the chemistry. We present here an experimental investigation of O-atom diffusion and reactivity on water ice. We explore the 6-25 $\mathrm{K}$ temperature range at sub-monolayer surface coverages. We derive the diffusion temperature law and observe the transition from quantum to classical diffusion. Despite of the high mass of O, quantum tunneling is efficient even at $6 \mathrm{~K}$. As a consequence, the solid-state astrochemistry of cold regions should be reconsidered and should include the possibility of forming larger organic molecules than previously expected.
\end{abstract}




\section{INTRODUCTION}

Nuclear decay or chemical reactions may be described as the crossing through a potential barrier by quantum tunneling, or crossing over the same barrier by thermal hopping (FIG. 1). Except for barrier-less reactions, increasing the temperature initiates the chemistry. Actually, the quantum tunneling regime (like in nuclear decay), and the thermal activation (like in chemistry) are usually separated by orders of magnitude in temperature. Theoretically, the balance between classical thermal motion and quantum tunneling is a very active subject, especially because it impacts solid state chemistry [15. It is well established that a critical temperature exists below which tunneling is dominant ([23],[14]). Experimentally, such an evidence is still missing in handbooks. Thanks to field ion microscopy, diffusion of single atoms on metals or on crystalline surfaces has been studied in detail for decades [12]. However, on amorphous surfaces, and especially on water ice substrates, the study of physisorbed light atoms presents enormous difficulties for any atomic microscopy technique that the field is still nearly unexplored. Yet, in the cold regions of the Universe, where temperatures are lower than $8 \mathrm{~K}$ [26], a rich chemistry is initiated on the surfaces of minuscule dust particles [30]. The species weakly bound to the surface are the pivotal media of this pristine chemistry, governed by the diffusion of reactive species [9]. So far, diffusion has only been partly explored experimentally for $\mathrm{H}$ atoms $([33],[22])$ and the role of amorphous structures in the diffusion properties is still an open question ([28, 34]). Nevertheless, the mobility of physisorbed species is key for the evolution of the molecular complexity [29]. If species like $\mathrm{O}$ atoms freeze out on the surface of the grains, the chemistry is governed by $\mathrm{H}$ additions, leading to numerous saturated species $\left(\mathrm{H}_{2} \mathrm{O}, \mathrm{NH}_{3}, \mathrm{CH}_{4}\right.$, which are chemical traps and end the chemical evolution). On the other hand, if other atoms (O, N, C...) are mobile enough at low temperature, other additions may open up the field of the observed molecular complexity reached in the first stages of star formation, and that could lead to the formation of the building blocks of life (amino-acids). Experimentally, very few studies have already involved physisorbed $\mathrm{O}$ atoms ([11,,[17],[32]) whereas theoretically, calculations exist for ordered substrates such as graphite $([3],[21])$. On cold surfaces, ozone reactivity has been the subject of experimental investigations for astrochemical ([24],[27]) and atmospheric

purposes [16]. Formation of $\mathrm{O}_{2}$ and $\mathrm{O}_{3}$ on amorphous silicates has been addressed recently [18] and will also be the subject of one of our future papers. We present here experimental 


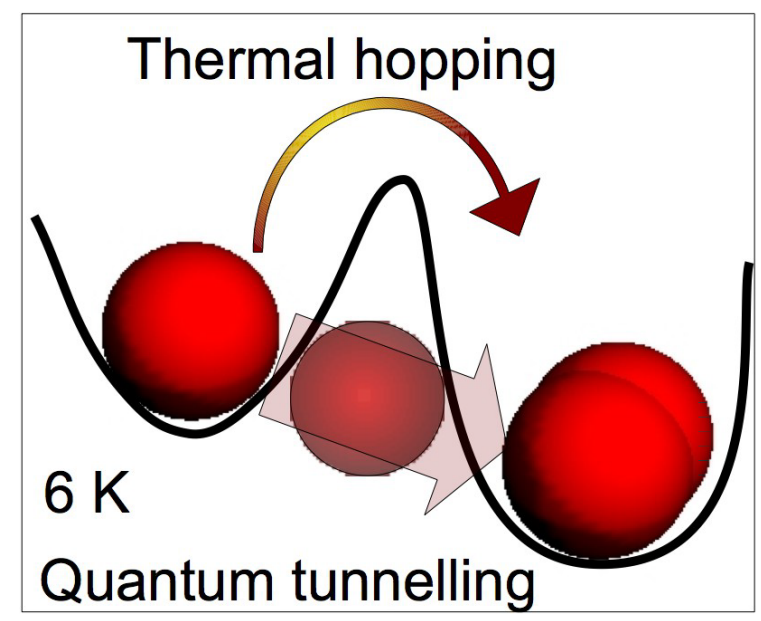

FIG. 1. Cartoon of thermal motion of an oxygen atom, vs quantum tunneling. Thermally-induced diffusion follows the regular exponential Arrhenius Law with T, quantum diffusion does not.

evidences of tunneling of physisorbed $\mathrm{O}$ atoms on different substrates (amorphous and crystalline water ice) via the study of ozone formation, and discuss the role of the morphology of the substrate in quantum diffusion process.

\section{EXPERIMENTS AND RESULTS}

Experiments have been performed using the FORMOLISM set-up (described elsewhere [1, [7]), an ultra-high vacuum (UHV) chamber coupled to a triply differentially pumped O beam aimed at the temperature controlled water ice samples. O atoms are obtained by dissociating $\mathrm{O}_{2}$ gas in a microwave discharge. The dissociation fraction $\mu$ is $71 \%$. It corresponds to depositing $3 \mathrm{O}_{2}$ molecules and $14 \mathrm{O}$ atoms. We have checked that atoms and molecules relax before adsorption by scanning the kinetic energy of the ionizing electrons of the mass spectrometer head intercepting the beam [8]. We detected no species with residual internal energy, i.e., having a ionization threshold below that of the ground state. No $\mathrm{O}_{3}$ was present in the beam either. The beam flux was calibrated using TPD (temperatureprogrammed-desorption) by determining the $\mathrm{O}_{2}$ exposure time required to saturate the $\mathrm{O}_{2}$ monolayer (ML) [25]. In this work, the exposures are expressed in terms of $\mathrm{O}_{2}$ units, which means that $1 \mathrm{ML}$ may also represent 2 layers of $\mathrm{O}$ atoms or 0.66 layers of pure ozone. The compact amorphous solid water (ASW) substrate was grown by vapor deposition on a $110 \mathrm{~K}$ substrate. We have also studied crystalline ice, made from an ASW substrate annealed up 


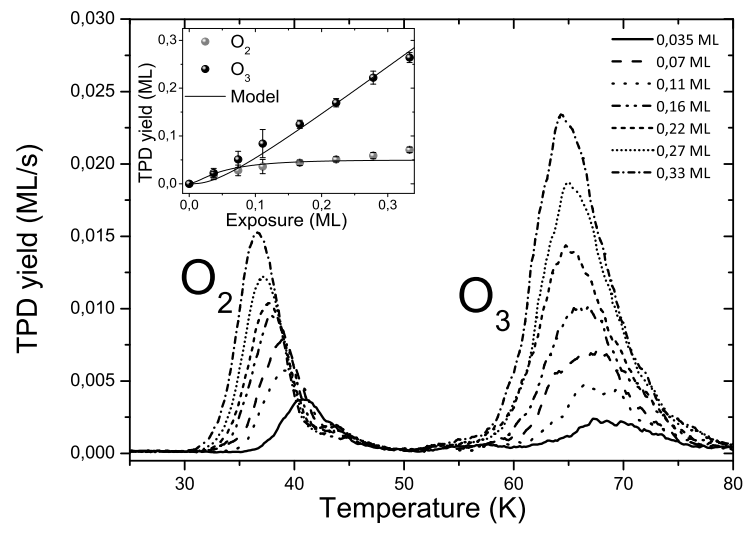

FIG. 2. TPD of $\mathrm{O}_{2}$ and $\mathrm{O}_{3}$ after deposition of various doses (0.04 - $\left.0.4 \mathrm{ML}\right)$ of $\mathrm{O} / \mathrm{O}_{2}$ mixture on ASW ice held at $10 \mathrm{~K}$. Inset: Areas of the TPD peaks (in ML/s) vs exposed $\mathrm{O} / \mathrm{O}_{2}$ dose.

to the phase transition temperature around 140K [25], and a "porous" ice template constituted of 1.0ML overlayer of porous water ice deposited at 10K over an ASW substrate. This substrate has no pores, but is topologically disordered and, particularly, presents already deep adsorption sites [13].

In all the experiments the substrate is heated steadily $(10 \mathrm{~K} / \mathrm{min})$ at the end of each deposition phase. Prior to each experiment, the sample is annealed to $100 \mathrm{~K}$ in order to stabilize the surface morphology before subsequent heating-cooling runs between 6.5 and $90 \mathrm{~K}$.

FIG. 2 shows the results of experiments performed by varying the $\mathrm{O} / \mathrm{O}_{2}$ doses. Two desorption peaks are present: $\mathrm{O}_{2}$ desorption occurs between $35 \mathrm{~K}$ and $50 \mathrm{~K}$, and the ozone desorption is observed between $55 \mathrm{~K}$ and $75 \mathrm{~K}$ (directly, or via the $\mathrm{O}_{2}^{+}$fragments). We observe, at any coverage or temperature, ozone formation by depositing $\mathrm{O}$ and $\mathrm{O}_{2}$ mixtures on ASW at $10 \mathrm{~K}$. $\mathrm{O}$ desorption is never observed. The shapes and positions of the $\mathrm{O}_{3}$ peaks are the same as those of $\mathrm{O}_{3}$ deposited from the gas phase, and are only coverage dependent. We can thus exclude any second order desorption effects, like it should be if $\mathrm{O}_{3}$ were formed on the onset of or during desorption. The circles in the inset of FIG. 2 represent the area under the TPD peaks as a function of deposited dose. The $\mathrm{O}_{3} / \mathrm{O}_{2}$ ratio increases with coverage, $\mathrm{O}_{2}$ reaches rapidly an almost steady state while $\mathrm{O}_{3}$ raises quite linearly. The solid lines in the inset represent the model results (see below). These experiments suggest that the reactivity of 
pure oxygen species is limited to two reactions:

$$
\begin{gathered}
\mathrm{O}+\mathrm{O} \rightarrow \mathrm{O}_{2} \quad R 1 \\
\mathrm{O}+\mathrm{O}_{2} \rightarrow \mathrm{O}_{3} \quad R 1
\end{gathered}
$$

The $\mathrm{O}+\mathrm{O}_{3} \rightarrow 2 \mathrm{O}_{2}$ reaction seems to be not competitive with the others, it would not be possible to obtain an almost pure $\mathrm{O}_{3}$ sample with increasing of the coverage. The two reactions $\mathrm{R} 1$ and $\mathrm{R} 2$ may arise from direct reactions between an impinging atom and an adsorbed species (Eley-Rideal mechanism, ER), or may occur by diffusion of the species on the surface (Langmuir-Hinshelwood mechanism, LH). It is also possible that the Hot Atom Mechanism (HAM) could have a role. Up to now HAM has been studied in conditions very different from ours: metallic surfaces, atoms with energy larger than $0.5 \mathrm{eV}$ or light atoms (H or D) ([31], 10] and references therein). The HAM is an initial diffusion of the impinging atoms due to their residual gas phase kinetic energy. We can consider this mechanism an extension of the ER mechanism as it occurs during the accommodation phase. In the case of $\mathrm{O}$ atoms, the kinetic energy of the beam is around $300 \mathrm{~K}$ and the sticking efficiency is more than $90 \%$, which indicates a good transfer of energy to the water ice substrate. The binding energy is about $1000 \mathrm{~K}$, thermal accommodation occurs in a few site jumps, since the atoms that stick have lost about 300K of the total energy upon their first impact. In what follows, we will consider the HAM mechanism included in the ER mechanism, but with an enhanced cross section $(\times 3)$. The ER mechanism happens between a gas phase reactant and a surface reactant, it is by construction not sensitive to the surface temperature and its efficiency depends on the coverage only. On the contrary, the LH mechanism (as well as diffusion) depends on the temperature of the surface. Therefore, during a TPD when temperature increases, this mechanism could lead to the formation of other $\mathrm{O}_{3} / \mathrm{O}_{2}$ molecules. We have attempted to check this possibility by following the evolution of $\mathrm{O}_{3}$ infrared absorption band intensity from $6.5 \mathrm{~K}$ to $35 \mathrm{~K}$. Because of a high detection limit (0.3 ML) this method could only be applied to the highest coverage experiment in Fig. 1, and even for this experiment the signal to noise is too low to provide strong constraints (not shown). Within the experimental uncertainties the $\mathrm{O}_{3}$ infrared band does not vary during the TPD for the high coverage experiment, except at the temperature above which ozone begins to come off the surface $(\sim 55 \mathrm{~K})$. This demonstrates that at least some $\mathrm{O}_{3}$ forms at deposition, and the results are consistent with the theory that thermally-induced diffusion during the TPD is a 


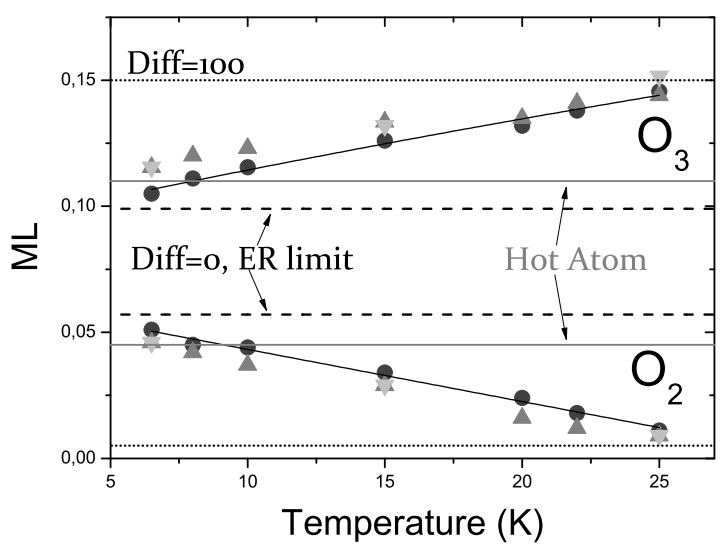

FIG. 3. $\mathrm{O}_{2}$ and $\mathrm{O}_{3}$ TPD peak areas after deposition of $0.21 \mathrm{ML}$ of $\mathrm{O} / \mathrm{O}_{2}$ mixture $(\mu=71 \%)$ on 3 substrates: crystalline ice (circles), non-porous ASW ice (triangles pointing up), and porous water ice (triangles pointing down). Solid lines: model results. Dashed and dotted lines: $\mathrm{O}_{2}$ (lower part) or $\mathrm{O}_{3}$ (upper part) amount in case of no diffusion (ER limit) and $\mathrm{k}=100 \mathrm{~s}^{-1}$. Dash-dotted line: model results with HAM and no diffusion.

secondary effect compared to diffusion and reactions at the deposition temperature.

To understand if diffusion effectively plays a role in the $\mathrm{O}_{3} / \mathrm{O}_{2}$ formation, we have performed a second set of experiments in which we varied the deposition temperature of the substrate and the morphology of the water substrate itself, but fixing the initial $\mathrm{O} / \mathrm{O}_{2}$ dose (coverage). As shown in FIG. 3 we observe that the $\mathrm{O}_{3} / \mathrm{O}_{2}$ ratio increases with the temperature of the substrate. The evolution as a function of the exposure temperature indicates that the temperature of the surface during irradiation is a key parameter. In FIG. 3 we show the $\mathrm{O}_{3}$ and $\mathrm{O}_{2}$ yields for different temperatures and for three different ice substrates (ASW ice, crystalline ice, and ASW ice coated with 1ML of porous water ice). There are differences between the three substrates, but they can be considered secondary if compared to the global trend. Even if the temperature dependence seems to be slow, at $25 \mathrm{~K}$ the $\mathrm{O}_{3}$ amount raises by $50 \%$ with respect to that at $6.5 \mathrm{~K}$, while in the same temperature range the $\mathrm{O}_{2}$ yield decreases by $75 \%$. During the TPD, the adsorbates follow the same thermal history, and should produce the same results. Therefore, the differences should originate at the time of the deposition phase. ER and HAM mechanisms are independent of the surface temperature, thus the evolution of the $\mathrm{O}_{3} / \mathrm{O}_{2}$ ratio is due to diffusion processes.

Basically, the $\mathrm{O}_{3} / \mathrm{O}_{2}$ balance is due to the diffusion of $\mathrm{O}$ atoms. If the diffusion is 
extremely fast, each newly adsorbed atom scans the surface until it reacts with $\mathrm{O}_{2}$ to form $\mathrm{O}_{3}$. If no $\mathrm{O}_{2}$ is present, $\mathrm{O}$ has to wait for another $\mathrm{O}$ atom to form $\mathrm{O}_{2}$, which in turn will be transformed into $\mathrm{O}_{3}$ via another incoming and mobile atom. Therefore, almost all the $\mathrm{O}$ atoms and $\mathrm{O}_{2}$ molecules are transformed into $\mathrm{O}_{3}$ molecules. On the contrary, if the diffusion is slow, one $\mathrm{O}$ atom has not enough time to scan the surface to meet an $\mathrm{O}_{2}$ molecule before another $\mathrm{O}$ comes. In this case $\mathrm{O}$-atoms accumulate until the probability for an $\mathrm{O}$ atom to meet another $\mathrm{O}$-atom raises, and finally $\mathrm{O}_{2}$ formation is favoured. In summary, FIG. 2 shows that an incoming $\mathrm{O}$-atom is more likely to find $\mathrm{O}_{2}$ molecules as the coverage increases. In FIG. 3 we show the increase of the $\mathrm{O}_{3} / \mathrm{O}_{2}$ ratio with deposition temperature which demonstrates the increase of diffusion processes with temperature.

\section{MODEL AND DISCUSSION}

As discussed in the previous section, only two reactions ( $\mathrm{R} 1$ and $\mathrm{R} 2$ ) occur on the surface via ER (HAM) or LH mechanisms. We have modeled our experiments adapting a classical set of differential equations [20] (see supplementary material for details). We assume a diffusion-limited reactivity (no reaction barrier), including both ER and LH mechanisms (same efficiency), or HAM which is estimated by enhancing the ER mechanism, Anyway, Oatoms are of mass high enough to be able to transfer in each single collision a relevant fraction of their kinetic energy, and so in few jumps O-atoms are thermalized. Possible adjustments due to thermally-induced diffusion during TPDs are also considered in order not to exclude the possibility of incomplete reactions during the exposure phase, even if we already noticed that it leads minor effects. There is only one physical free parameter to adjust, $k$, which represents the effective surface diffusion. Therefore $\mathrm{O}_{3} / \mathrm{O}_{2}$ ratio at $25 \mathrm{~K}$ is 45 times bigger than that at $6.5 \mathrm{~K}$. Using only one adjustable parameter $k$ we reproduce perfectly all our data sets (solid lines in FIG. 2 and FIG. 3). Other alternative scenarios (barrier to reaction, low diffusivity, pure LH or ER mechanisms, and HAM) have been tested without the same success. In FIG. 3 the boundaries of the ER mechanism and HAM are represented by two constant lines. These limits are not sensitive to the surface temperature. Actually, it is possible to fit the coverage dependency with several different hypothesis (or parameters), but it is not possible to have both temperature and coverage dependencies satisfied at once. Plain circles of FIG. 4 show the diffusion law for $\mathrm{O}$ as a function of the temperature, obtained 
from the change in the balance of $\mathrm{O}_{2}$ and $\mathrm{O}_{3}$ production, assuming a diffusion dominated process. The diffusion coefficient increases by a factor of 50, but the logarithm scaling of the figure tends to flatten this aspect of the experimental results. The trend is somehow surprising because the measured diffusion barrier does not follow an Arrhenius law. Empty circles in FIG. 4represent a typical Arrhenius law with a diffusion barrier of 450K. Therefore, a pure thermal diffusion does not represent well our data. The diffusion is better simulated using the quantum tunneling of a square barrier, as described in Messiah's book [23]. We use two physical parameters: the width $a$ and the height of the barrier $E_{a}$. As already described [6], diffusion includes two components, quantum tunneling that dominates at low temperatures, and thermal diffusion predominant at higher temperatures. The arrow in FIG. 4 represents the critical temperature where the transition occurs. In our experimental data we observe that the quantum to classical regime occurs at around $20 \mathrm{~K}$. We find the best results for $a=0.70 \pm 0.05 \AA$ and $E_{a}=520 \pm 60 \mathrm{~K}$. These two parameters have different effects on the diffusion curves as shown in FIG. 4 (dashed and dotted lines). The pinstriped region in FIG. 4 represents the validity zone of the solution we found, since it is possible to partly compensate for the variation of the height of the barrier by changing its width. Adopting a square barrier is an extreme assumption, and the apparent low value of the height we found may be due to this unrealistic shape.

The main results of our study are the following: first, O-atom diffusion is governed by quantum tunneling up to 20K. Our experiments show nicely the transition from the quantum world to the classic world. The thickness of the barrier $(0.7 \AA)$ may also be due to the amorphous nature of the ice. Indeed, one can consider that the diffusion on amorphous surface is dominated by the fastest jumps between adsorption sites, and that the diffusion barriers limiting the diffusion are the weakest ones [19]. Therefore, the apparent low value of width is probably an estimation of the lower limit of the distribution of barrier widths of this disordered substrate. We want now discuss the signification of a width barrier of $0.7 \AA$. On water ice the mean distance of two adsorption sites is $3 \AA$, while the de Broglie wavelength $\left(\lambda_{b}\right)$ associated to $\mathrm{O}$ atoms varies from $2.8 \AA$ at $6.5 \mathrm{~K}$ to $1.5 \AA$ at $20 \mathrm{~K}$. It corresponds to the typical size where quantum effects are important. We can compare the $3 \AA$ mean distance with the double (2 atoms diffusion-reaction) of $\lambda_{b}$. For temperatures lower than $22 \mathrm{~K}$, quantum effects should thus be important. This is actually what we observe. The slightly lower value ( 0.7 instead of $1 \AA$ guessed in the literature), could also be due to the 


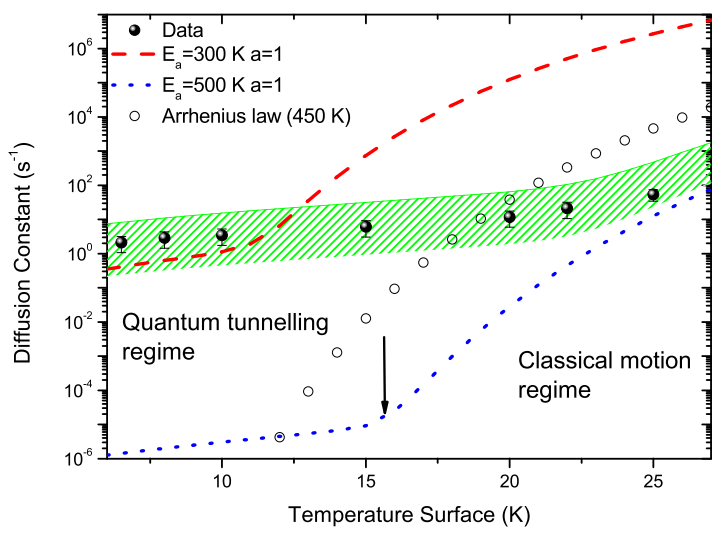

FIG. 4. Diffusion coefficient of O atoms on amorphous ice as function of the surface temperature. Plain circles: experimental values. Open circles: Arrhenius law $\left(\mathrm{E}_{a}=450 \mathrm{~K}\right)$. Dashed and dotted line: best fit of the diffusion using both quantum and thermal diffusion following [18 $(a=1 \AA$, $\left.\mathrm{E}_{a}=500 \mathrm{~K}-300 \mathrm{~K}\right)$. Pinstripe zone: $\mathrm{a}=0.7 \pm 0.05 \AA, \mathrm{E}_{a}=520 \mathrm{~K} \pm 60 \mathrm{~K}$. The arrow indicates the point on the dotted curve where the quantum to classical transition occurs.

disordered nature of the surface that distribute distances between adjacent adsorption sites, and so reduces some of them. Therefore cold $\mathrm{O}$ atoms would easily overlap 2 neighbouring sites.

Secondly, comparing the different morphologies of the ice allows us to conclude that the topological disorder of the substrate does not deeply affect the diffusion regime. This probably means that the lower limits of the distributions of barrier widths are not too different from each other for the substates considered here. However, we find that the diffusion on the crystalline surface is faster than that on amorphous ice. This could be explained as follows: i) the wave packet describing the adsorbed atom diffuses more quickly in the periodic potential of a crystalline surface than in the amorphous one [19]. ii) Another study [34] showed that the diffusion and reactive properties are greatly changed by the occurrence of deep and shallow sites. In our case, we find that the presence of deep sites ("porous" substrate) does not reduce significantly the effective diffusion. The trapping sites may force one $\mathrm{O}$ atom to stay in one adsorption site, but they cannot prevent another atom from reaching it, especially if tunneling dominates.

Third conclusion, we find that the diffusion of oxygen at $10 \mathrm{~K}$ is $k=5 s^{-1}$, which corresponds 
in classical Arrhenius formalism to thermal diffusion barrier of 300K. The diffusion is faster than previously guessed by astronomers (diffusion barrier of 400K [30] or even 900K [5]. However, it is not very far from that of $\mathrm{O}$ diffusion in matrices, 240 $\pm 80 \mathrm{~K}$ [2], which could be considered an upper value for surface diffusion. Based upon arguments of polarizability scaling [29], and the mean value of [22], we would expect a value of $275 \pm 30 \mathrm{~K}$, which is consistent with what is derived here. From our model we can indirectly conclude that the maximal barrier height for the $\mathrm{O}_{2}+\mathrm{O}$ reaction is $190 \mathrm{~K}$. Actually, if the barriers were higher, there would be noticeable discrepancies in the $\mathrm{O}_{2} / \mathrm{O}_{3}$ ratio, which we do not observe. It is probably even smaller as estimated in a previous study [2]. Implications for solid state astrochemistry are of major importance. It was usually thought that the chemistry was mostly driven by $\mathrm{H}$ diffusion, and therefore final products were mostly hydrogen saturated species such as $\mathrm{H}_{2} \mathrm{O}, \mathrm{NH}_{3}, \mathrm{CH}_{4}, \mathrm{CH}_{3} \mathrm{OH}$. We can affirm now that $\mathrm{O}$ addition chemistry is competitive with $\mathrm{H}$ additions, because of the comparable budget of $\mathrm{O}$ atoms and $\mathrm{H}$ atoms in dense and UV protected interstellar environments [4]. Furthermore, if we scale the diffusion for other atoms such as $\mathrm{C}$ and $\mathrm{N}$, on the basis of their polarizability alone - the main parameter for physisorption - there is a reasonable range of temperatures $(\leq 15 \mathrm{~K})$ where mobility of $\mathrm{O}, \mathrm{C}$ and $\mathrm{N}$ is activated. For this reason, the production of $\mathrm{O}, \mathrm{C}$, and $\mathrm{N}$ bearing molecules can grow, avoiding saturated chemical traps. This could also be one of the source of complex non-volatile organic compounds observed in meteorites, such as amino-acids.

We acknowledge the support of the national PCMI programme founded by CNRS, the Conseil Regional dIle de France through SESAME programmes (contract I-07597R). MM acknowledges LASSIE, a European FP7 ITN Communitys Seventh Framework Programme under Grant Agreement No. 238258. MA thanks COST ACTION CM0805.We also thank the unknown referees for the fruitful comments and suggestions that helped make a better paper.

\section{SUPPLEMENTARY MATERIALS: MODEL. SET OF DIFFERENTIAL EQUA- TIONS USED TO ANALYZE THE EXPERIMENTAL DATA.}

For the sake of clarity, the equation are detailed in two sets, even if they are solved simultaneously. One corresponds to the diffusion (LH mechanism) and another to the ER 
mechanism. The LH mechanism is described as follows

$$
\begin{aligned}
\frac{d O}{d t} & =4 k O O-k O O_{2} \\
\frac{d O_{2}}{d t} & =2 k O O-k O O_{2} \\
\frac{d O_{3}}{d t} & =k O O_{2}
\end{aligned}
$$

$\mathrm{O}_{x}$ is the fraction of occupied sites of the species $x$ (it is equal to one when the coverage is $1 \mathrm{ML}=10^{15} \mathrm{~cm}^{-2}$ ), and $k$ is the diffusion parameter (in $\mathrm{s}^{-1}$ ). The increase of the $\mathrm{O}_{3}$ population is proportional to the density of reactants times the diffusion coefficient. EleyRideal mechanism is described as follows:

$$
\begin{aligned}
\frac{d O}{d t} & =2 \mu \phi\left(1-2 O-O_{2}\right)-(1-\mu) \phi O \\
\frac{d O_{2}}{d t} & =(1-\mu) \phi(1-O)-2 \mu \phi\left(O_{2}-O\right) \\
\frac{d O_{3}}{d t} & =(1-\mu) \phi O+2 \mu \phi O_{2}
\end{aligned}
$$

$\phi$ is the flux and $\mu$ is the dissociation rate. The cross section is supposed to be as large as the adsorption site area (so equal to 1, not shown), which can be considered as an upper value. Nevertheless, the ER mechanism does not take a large part in the calculations, especially at low coverages. The time is integrated for the duration of the experimental exposure.

* email adress: marco.minissale@obspm.fr

[1] L. Amiaud, F. Dulieu, J.-H. Fillion, A. Momeni, and J. L. J. L. Lemaire, JCP 127, 094702 (2007).

[2] A. V. Benderskii and C. A. Wight, JCP 104, 85 (1996).

[3] H. Bergeron, N. Rougeau, V. Sidis, M. Sizun, D. Teillet-Billy, and F. Aguillon, JCP A 112, 11921 (2008).

[4] P. Caselli, O. Stantcheva, T. Shalabiea, V. Shematovich, and E. Herbst, P\&SS 50, 1257C (2002).

[5] S. Cazaux, V. Cobut, M. Marseille, M. Spaans, and P. Caselli, A\&A A74, 2010 (522).

[6] S. Cazaux and A. Tielens, ApJ 604, 222 (2004).

[7] E. Congiu, H. Chaabouni, C. Laffon, P. Parent, S. Baouche, and F. Dulieu, JCP 137, 054713 (2012). 
[8] E. Congiu, E. Matar, L. E. Kristensen, F. Dulieu, and J. L. Lemaire, MNRAS 397L, 96C (2009).

[9] H. M. Cuppen and E. Herbst, ApJ 668, 294 (2007).

[10] L. C. Dinger, A. and J. Kuppers, J. Chem. Phys. 114, 12, 5338 (2001).

[11] F. Dulieu, L. Amiaud, E. Congiu, J.-H. Fillion, E. Matar, A. Momeni, V. Pirronello, and J. L. Lemaire, A\&A 512, A30 (2010).

[12] G. Ehrlich, Surf. Sci. 299, 628 (1994).

[13] J.-H. Fillion, L. Amiaud, E. Congiu, F. Dulieu, A. Momeni, and J.-L. Lemaire, PCCP 11, 4396 (2009).

[14] M. J. Gillan, JP C: Solid State Phys. 20, 3621 (1987).

[15] T. P. M. Goumans and S. Andersson, MNRAS 406, 2213 (2010).

[16] C. Janssen and B. Tuzson, JPC A 114, 9709 (2010).

[17] D. Jing, J. He, J. Brucato, A. De Sio, L. Tozzetti, and G. Vidali, ApJ 714L, 9J (2011).

[18] D. Jing, J. He, J. R. Brucato, G. Vidali, L. Tozzetti, and A. De Sio, ApJ 756, 98 (2012).

[19] L. Karssemeijer, A. Pedersen, H. Jonsson, and H. Cuppen, PCCP 134, 084504 (2012).

[20] N. Katz, I. Furman, O. Biham, V. Pirronello, and G. Vidali, ApJ 522, 305 (1999).

[21] R. Larciprete, P. Lacovig, S. Gardonio, A. Baraldi, and S. Lizzit, JPC C 116, 9900 (2012).

[22] E. Matar, E. Congiu, F. Dulieu, A. Momeni, and J. L. Lemaire, A\&A 492, L17 (2008).

[23] A. Messiah, Quantum Mechanics (Wiley Amsterdam, North Holland,, 1973).

[24] H. Mokrane, H. Chaabouni, E. Accolla, M. andCongiu, F. Dulieu, M. Chehrouri, and J. L. Lemaire, ApJL 705, L195 (2009).

[25] J. A. Noble, E. Congiu, F. Dulieu, and H. J. Fraser, MNRAS 421, 768779 (2012).

[26] L. Pagani, A. Bacmann, S. Cabrit, and C. Vastel, A\&A 467, 179 (2007).

[27] C. Romanzin, S. Ioppolo, H. M. Cuppen, E. F. van Dishoeck, and H. Linnartz, JCP 134, 084504 (2011).

[28] R. Smoluchowski, JPC 87, 4229 (1983).

[29] A. G. G. M. Tielens and L. J. Allamandola, in Cool Interstellar Physics and Chemistry. (Pan Stanford Publishing Pte Ltd: Singapore, 2011).

[30] A. G. G. M. Tielens and W. Hagen, A\&A 114, 245 (1982).

[31] M. Tomellini, Physica A 392, 875T (2013).

[32] M. D. Ward and S. D. Price, ApJ 741, 121 (2011). 
[33] N. Watanabe, Y. Kimura, A. Kouchi, T. Chigai, T. Hama, and V. Pirronello, ApJL 714, L233 (2010).

[34] A. Wolff, I. Lohmar, J. Krug, Y. Frank, and B. O., Phys. Rev. E 81, 061109 (2010). 